\title{
Deconvolution of Atomic Photoabsorption Spectra: a match between theory and experiment
}

\author{
T. N. Chang and Y. X. Luo \\ Department of Physics and Astronomy, University of Southern California Los Angeles, CA \\ 90089-0484, USA \\ H. S. Fung and T. S. Yih \\ Department of Physics, National Central University, Chung-Li, TAIWAN \\ ROC 32054
}

We present in this paper an extension of a recently proposed deconvolution procedure to compare directly the theoretical and experimental spectrum of a doubly excited ultra-narrow and nearly symmetric resonance in atomic photoabsorption. Our discussion is based on a set of analytical relations in terms of the variations of i) the ratio between the resonance width $\Gamma$ and the experimental energy resolution $\Omega$ in the limit when $\Gamma / \Omega \ll 1$ and ii) the column density $n l$ of the media in a photoabsorption experiment.

PACS number(s): 32.80Fb, 32.70.Jz, 32.80.Dz.

\section{Introduction}

In an attempt to estimate the width of a narrow isolated doubly excited resonance from measured spectra in the absence of an ultrahigh-energy resolution, Fang and Chang [1] have recently proposed a deconvolution procedure which enables a direct extrapolation to infinite energy resolution using a set of explicit analytical relations in terms of the ratio $R$ of the resonant width $\Gamma$ and the experimental energy resolution $\Omega$ in the limit of $R=\Gamma / \Omega \ll 1$. This procedure applies well for a photoionization experiment when the photoion and/or the photoelectron are measured directly. Unlike the photoionization, the resonant spectra in a photoabsorption experiment is determined by detecting the light attenuation through a medium. It is known that the measured cross section is significantly affected by the column density $n l$ of the medium and the experimental monochromator (or slit) function $\mathcal{F}[2]$.

The photoabsorption cross-section $\sigma^{P a}(E)$ at a photon energy $E$ is determined experimentally using the Beer-Lambert law,

$$
I(E)=I_{o}(E) e^{-n l \sigma^{P a}(E)} .
$$

where $I_{o}$ is the intensity of the incident light, $I$ is the attenuated intensity of the transmitted light, and $n l$ is the column density. At a photon energy $E, I_{o}$ and $I$ can be expressed in terms of the slit function $\mathcal{F}$ centered at $E$ and characterized by an energy resolution $\Omega$, i.e.,

$$
I_{o}(E)=\int i_{o} \mathcal{F}\left(E^{\prime}-E ; \Omega\right) d E^{\prime}
$$

and

$$
I(E)=\int i_{o} \mathcal{F}\left(E^{\prime}-E ; \Omega\right) e^{-n l \sigma(E)} d E^{\prime},
$$


where $\sigma$ is the cross section at an infinite energy resolution (i.e., at $\Omega=0$ ). From Eqs. (1$3)$, the measured resonance structure in a photoabsorption experiment is represented by a convoluted spectrum in the form of

$$
\sigma^{P a}(E)=-\frac{1}{n l} \ln \left(\int \mathcal{F}\left(E^{\prime}-E ; \Omega\right) e^{-n l \sigma\left(E^{\prime}\right)} d E^{\prime}\right) .
$$

As expected, when $n l \rightarrow 0$, the cross section takes the same form of the photoionization given by Eq. (3) of Ref. [1], i.e., $\sigma^{P a} \rightarrow \sigma^{P i}$, where

$$
\sigma^{P i}(E)=\int \sigma\left(E^{\prime}\right) \mathcal{F}\left(E^{\prime}-E ; \Omega\right) d E^{\prime}
$$

The slit function $\mathcal{F}$ may be approximated at the center by a Gaussian distribution $\mathcal{G}$ and modified at its tail by a Lorentzian distribution $\mathcal{L}$. It can be expressed by a weighted combination of $\mathcal{G}$ and $\mathcal{L}[1,3]$, i.e.,

$$
\mathcal{F}\left(E ; \Omega, w_{g}, w_{\ell}\right)=w_{g} \mathcal{G}(E ; \Omega)+w_{\ell} \mathcal{L}(E ; \Omega),
$$

where the sum of $w_{g}$ and $w_{\ell}$ equals one. ( $\mathcal{G}$ and $\mathcal{L}$ are given explicitly by Eq. (4) of Ref. [1].) There is no well established general procedure to determine the weighting factors $w_{g}$ and $w_{\ell}$ experimentally in the absence of ultrahigh energy resolution. Based on the analytical relations discussed in the next section, we shall propose a procedure leading to the determination of $w_{g}$ and $w_{\ell}$.

The density effect in photoabsorption can be easily illustrated by the variation of the convoluted spectra of an isolated resonance with changing $n l$ and $\Omega$. For simplicity, we will limit our discussion using a Fano-type of resonance described by an asymmetry parameter $q$ and the smoothly varying background cross section $\sigma_{b}$, i.e., [4]

$$
\sigma(E)=\sigma_{b} \frac{(q+\epsilon)^{2}}{1+\epsilon^{2}}
$$

where the reduced energy $\epsilon=\left(E-E_{r}\right) /\left(\frac{1}{2} \Gamma\right)$ is defined in terms of the energy $E_{r}$ and the width $\Gamma$ of the resonance. The cross section $\sigma$ is expected to reach its peak value $\sigma_{\max }=\sigma_{b}\left(1+q^{2}\right)$ and a zero at energies

$$
E_{\max }=E_{r}+\frac{1}{2}(\Gamma / q) \text { and } E_{\min }=E_{r}-\frac{1}{2}(\Gamma q)
$$

respectively. Figure 1 presents a number of selected convoluted photoionization spectra using Eq. (5) with $R$ ranging from $1 / 10$ to $1 / 25$ and a slit function $\mathcal{F}$ represented either by a Gaussian distribution $\mathcal{G}$ or a Lorentzian distribution $\mathcal{L}$. These spectra correspond to a fictitious resonance derived from Eq. (7) with $E_{r}=2.110 \mathrm{Ry}, \sigma_{b}=1.0 \mathrm{Mb}, q^{2}=2500$, and $\Gamma=10^{-6}$ Ry. As expected, for a given ratio $R$, the peak cross section $\sigma_{\max }$ corresponding to the spectrum convoluted using Gaussian distribution is substantially higher than the one using Lorentzian distribution. The density effect in photoabsorption measurement is unambiguous demonstrated by the substantial reduction in peak cross sections shown in Fig. 2 when the same spectrum is convoluted using Eq. (4) with a column density $n l=$ $0.001 \mathrm{Mb}^{-1}$. 


\section{Peak Cross Sections}

For an ultra-narrow and nearly symmetric resonance, the peak cross section $\sigma_{\max }$ is very well approximated by the cross section at $E=E_{r}$, i.e., $\sigma_{\max } \approx \sigma\left(E_{r}\right)$, since, from Eq. (8), the energy corresponding to the peak cross section, i.e., $E_{\max }$, equals approximately the resonant energy $E_{r}$ as the difference $E_{\max }-E_{r}$ is substantially smaller than the resonance width, i.e., as $q^{2} \gg 1$ and $\frac{\Gamma}{q} \ll \Gamma$.

In a photoionization experiment,

$$
\sigma_{\text {max }}^{P i} \cong \int \sigma\left(E^{\prime}\right) \mathcal{F}\left(E^{\prime}-E_{r} ; \Omega\right) d E^{\prime}
$$

Although Eq. (5) is not in general integrable for an arbitrary energy E, Eq. (9) can be integrated analytically. For a Lorentzian distribution,

$$
\sigma_{\max }^{L}=\sigma_{b}\left(1+q^{2} R\right) /(1+R)
$$

and for a Gaussian distribution,

$$
\sigma_{\max }^{G}=\sigma_{b}\left(1+\pi^{1 / 2}\left(q^{2}-1\right)\right) R e^{R^{2}} F_{c}(R),
$$

where $F_{c}(x)=1-(2 / \sqrt{\pi}) \int_{0}^{x} e^{-y^{2}} d y$ is the complementary error function. For a nearly symmetric ultra-narrow resonance, such as the fictitious resonance shown in Figs. 1 and 2, our calculation shows that the approximate $\sigma_{\max }$ at $E=E_{r}$, derived from Eqs. (10) and (11), are within $0.05 \%$ of the exact peak cross sections determined from the numerically calculated spectra using Eq. (5). In a photoabsorption experiment, the peak cross section $\sigma_{\max }$ can also be approximated similarly from Eq. (4) as

$$
\sigma_{\max }^{p a} \cong-\frac{1}{n l} \ln \left(\int \mathcal{F}\left(E^{\prime}-E_{r} ; \Omega\right) e^{-n l \sigma\left(E^{\prime}\right)} d E^{\prime}\right)
$$

Figure 3 shows that the approximate peak cross sections $\sigma_{\max }$, represented by the nearly straight lines obtained from Eq. (12) are in close agreement with the exact peak cross sections at $R=\frac{1}{25}, \frac{1}{20}, \frac{1}{15}$ and $\frac{1}{10}$ for a number of column densities $n l$ derived directly from the numerically calculated convoluted spectra using Eqs. (4) and (5) for photoabsorption and photoionization (i.e., when $n l=0$ ), respectively.

In general, Eq. (12) can not be integrated analytically due to the exponential term $e^{-n l \sigma}$. However, when $e^{-n l \sigma}$ is expanded into an infinite series, each individual term becomes integrable and $\sigma_{\max }$ can be expressed in terms of a polynomial in $R$, i.e.,

$$
\sigma_{\max } \rightarrow \sigma^{P a}\left(E=E_{r}\right)=q^{2} \sigma_{b} X(R, n l),
$$

where

$$
X(R, n l)=\sum_{i=1}(-1)^{i+1} \xi_{i}(\rho) R^{i}
$$


and $\rho$ is a parameter given by

$$
\rho=n l q^{2} \sigma_{b} .
$$

For a Lorentzian distribution, the first few expansion coefficients are

$$
\begin{gathered}
\xi_{1}=1-\frac{1}{4} \rho+\frac{1}{16} \rho^{2}-\frac{5}{384} \rho^{3}+\frac{7}{3072} \rho^{4}-\cdots \\
\xi_{2}=1-\frac{1}{2} \rho+\frac{1}{4} \rho^{2}-\frac{3}{32} \rho^{3}+\frac{11}{384} \rho^{4}-\cdots \\
\xi_{3}=1-\frac{3}{4} \rho+\frac{9}{16} \rho^{2}-\frac{119}{384} \rho^{3}+\frac{141}{1024} \rho^{4}-\cdots \\
\xi_{4}=1-\rho+\rho^{2}-\frac{35}{48} \rho^{3}+\frac{41}{96} \rho^{4}-\cdots \\
\xi_{5}=1-\frac{5}{4} \rho+\frac{25}{16} \rho^{2}-\frac{545}{384} \rho^{3}+\frac{3175}{3072} \rho^{4}-\cdots
\end{gathered}
$$

and for a Gaussian distribution

$$
\begin{gathered}
\xi_{1}=\sqrt{\pi}\left(1-\frac{1}{4} \rho+\frac{1}{16} \rho^{2}-\frac{5}{384} \rho^{3}+\frac{7}{3072} \rho^{4}-\cdots\right) \\
\xi_{2}=2-\pi\left(\frac{1}{2} \rho-\frac{1}{4} \rho^{2}+\frac{3}{32} \rho^{3}-\frac{11}{384} \rho^{4}+\cdots\right) \\
\xi_{3}=\sqrt{\pi}\left(1-\frac{7}{4} \rho+\left(\frac{23}{48}+\frac{\pi}{3}\right) \rho^{2}-\left(\frac{47}{384}+\frac{\pi}{4}\right) \rho^{3}+\left(\frac{79}{3072}+\frac{\pi}{8}\right) \rho^{4}-\cdots\right) \\
\xi_{4}=\frac{4}{3}(1-\rho)-\pi\left(\rho-2 \rho^{2}+\left(\frac{47}{48}+\frac{\pi}{4}\right) \rho^{3}-\left(\frac{35}{96}+\frac{\pi}{4}\right) \rho^{4}+\cdots\right) \\
\xi_{5}=\sqrt{\pi}\left(\frac{1}{2}-\frac{71}{24} \rho+\left(\frac{307}{96}+\pi\right) \rho^{2}-\left(\frac{673}{768}+\frac{9 \pi}{4}\right) \rho^{3}+\left(\frac{20329}{92160}+\frac{37 \pi}{24}+\frac{\pi^{2}}{5}\right) \rho^{4}-\cdots\right) .
\end{gathered}
$$

Under a typical experimental condition, even at a fairly low column density $n l$, the parameter $\rho$ may be close to or greater than unity as $q^{2} \gg 1$. Consequently, the peak cross section $\sigma_{\max }$ can be estimated approximately from Eq. (13) only if $R$ is very small and the number of contributing $\xi_{i}$ terms is limited. More discussion will be given in section III. 


\section{Proposed Procedures}

\section{A. Determination of $w_{\ell}$ and $w_{g}$ in photoionization}

When $\Gamma \ll \Omega$ (or, $R \ll 1$ ), for a nearly symmetric resonance with $q^{2} \gg 1$, the observed $\sigma_{\max }=w_{\ell} \sigma_{\max }^{L}+w_{g} \sigma_{\max }^{G}$ can be expressed approximately according to Eqs. (10) and (11) as

$$
\sigma_{\max } \rightarrow\left(w_{\ell}+w_{g} \pi^{1 / 2}\right)\left(q^{2} \sigma_{b}\right) R
$$

or, $\sigma_{\max }$ varies linearly as functions of $1 / \Omega$. As a result,

$$
\left(w_{\ell}+w_{g} \pi^{1 / 2}\right)\left(q^{2} \Gamma \sigma_{b}\right)=S_{1},
$$

where $S_{1}$ is the slope determined experimentally by a plot of $\sigma_{\max }$ vs. $1 / \Omega$ according to Eq. (12). In addition, according to Eq. (17) of Ref. [1],

$$
\left(1.3282 w_{\ell}+1.9646 w_{g}\right)\left(q^{2} \Gamma \sigma_{b}\right)=S_{2},
$$

where $S_{2}$ is also a slope determined experimentally by a procedure detailed in Ref. [1]. Eqs. (27) and (28), together with $w_{\ell}+w_{g}=1$, offer an unambiguous procedure to determine the weighting factors $w_{\ell}$ and $w_{g}$ in a photoionization experiment.

\section{B. Determination of $q^{2} \sigma_{b}$ and $\Gamma$.}

Figure 4 shows that the approximate peak cross sections, $\sigma_{E=E_{r}}$, obtained from the analytical expression Eq. (13) remain in close agreement with the numerically calculated $\sigma_{E=E_{r}}$ from Eq. (12) and the exact $\sigma_{\max }$ from Eq. (4) for a value of $\rho=n l q^{2} \sigma_{b}$ as large as 1.25. Clearly, as $\rho$ increases to a value of 2.5 , Eq. (13) is no longer applicable.

Since $\Omega($ not $R$ ) is an experimentally measured variable, we shall now work with an alternative polynomial, in stead of the polynomial $X(R, n l)$, for $\sigma_{\max }$, i.e.,

$$
\sigma_{\max }=\left(q^{2} \sigma_{b} \Gamma\right) Y(\eta, n l)
$$

where $Y(\eta, n l)$ takes the form

$$
Y(\eta, n l)=\sum_{i=1}(-\Gamma)^{i-1} \xi_{i}(\rho) \eta^{i}
$$

and

$$
\eta=1 / \Omega
$$

Our proposed procedure starts with a best fit of the measured $\sigma_{\max }$ at a number of energy resolutions to an expression

$$
\sigma_{\max }(\eta ; n l)=\sum_{\mu=1} \alpha_{\mu}(n l) \eta^{\mu}
$$


By comparing Eq. (32) to Eq. (29), the fitted coefficients $\alpha_{\mu}$ for a given $n l$ is independent of $\Omega$. In addition, the ratio of two fitted values of $\alpha_{\mu}$ at two different column densities equals to the ratio of two $\xi_{\mu}$, i.e.,

$$
\frac{\alpha_{\mu}(n l)}{\alpha_{\mu}\left(n^{\prime} l^{\prime}\right)}=\frac{\xi_{\mu}(\rho)}{\xi_{\mu}\left(\rho^{\prime}\right)} .
$$

Since $\sigma_{\max }$ is a slowly varying function of $\eta$, a number of experimentally determined ratios between several pairs of $\alpha_{1}$ obtained at different column densities should be sufficient to fit adequately a value of $q^{2} \sigma_{b}$. With a best fitted $q^{2} \sigma_{b}$, the resonant width $\Gamma$ can be determined readily from Eq. (13) or (29).

\section{Results and Discussion}

Figure 5 presents the variation of the simulated photoabsorption peak cross sections $\sigma_{\max }$ as a function of $1 / \Omega$ at several column densities. It is derived from the convoluted spectra numerically calculated from Eq. (4) for a fictitious resonance with a width $\Gamma=5 \times 10^{-5} \mathrm{Ry}$, $q^{2}=400$, and $\sigma_{b}=0.015 \mathrm{Mb}$. Following the procedure outlined in section III.B, for each $n l$, a parameter $\alpha_{1}$ is first least-square fitted from Eq. (29). Second, from Eq. (30), we obtain a best fitted value of $5.42 \mathrm{Mb}$ and $5.54 \mathrm{Mb}$ for $q^{2} \sigma_{b}$ using the Lorentzian and Gaussian distribution, respectively. Finally, Eq. (13) leads to a width of $5.67 \times 10^{-5}$ Ry (Lorentzian) and $5.48 \times 10^{-5}$ Ry (Gaussian). The 10\% error introduced in this application is not unexpected due to values of $\rho$ which exceed unity for some of the column densities.

The deconvolution procedure proposed in this paper works best when $q^{2} \gg 1$. It clearly posts a difficult experimental challenge as it also requires simultaneously a small parameter $\rho=n l q^{2} \sigma_{b}$ when a small column density $n l$ may adversely reduce the signal to noise ratio in a photoabsorption experiment. In spite of this difficulty, the procedure proposed above offers a realistic possibility to take advantage of the density effect, in an attempt to determine experimentally the width of an ultra-narrow and nearly symmetric atomic resonance which can not be measured directly otherwise.

\section{Acknowledgments}

This work is partially supported by the National Science Council of Taiwan under contract NSC 88-2112-M008-011 (TSY) and by NSF Grant No. PHY9802557 (TNC).

\section{References}

[1] T. K. Fang and T. N. Chang, Phys. Rev. A57, 4407 (1998).

[2] R. D. Hudson and V. L. Carter, J. Op. Soc. Am. 58, 227 (1968); W. F. Chan, G. Cooper, and C. E. Brion, Phys. Rev. A44, 186 (1991).

[3] K. Schulz, G. Kaindl, M. Domke, J. D. Bozek, P. A. Heimann, A. S. Schlachter, and J. M. Rost, Phys. Rev. Lett. 77, 3086 (1996).

[4] U. Fano, Phys. Rev. 124, 1866 (1961). 


\section{Figure captions}

Figure 1. Convoluted photoionization spectra using Eq. (5) with $R$ ranging from $1 / 10$ to $1 / 25$.

Figure 2. Convoluted photoabsorption spectra using Eq. (4) with $R$ ranging from $1 / 10$ to $1 / 25$ and a column density $n l=0.001 \mathrm{Mb}^{-1}$.

Figure 3. Comparison between $\sigma_{\max }$ (nearly straight lines) obtained from Eq. (12) and the exact peak cross sections at $R=\frac{1}{25}, \frac{1}{20}, \frac{1}{15}$ and $\frac{1}{10}$ for a number of column densities $n l$ derived directly from the numerically calculated convoluted spectra using Eqs. (4) and (5) for photoabsorption and photoionization (i.e., when $n l=0$ ), respectively.

Figure 4. Comparison of the approximate peak cross sections, $\sigma_{E=E_{r}}$, obtained from the analytical expression Eq. (13), the numerically calculated $\sigma_{E=E_{r}}$ from Eq. (12) and the exact $\sigma_{\max }$ from Eq. (4).

Figure 5. Variation of simulated photoabsorption peak cross sections $\sigma_{\max }$ (from Eq. (4)) as a function of $1 / \Omega$ at column densities ranging from $0.07 \mathrm{Mb}^{-1}$ to $0.23 \mathrm{Mb}^{-1}$. (Only Lorentzian data are shown.) 

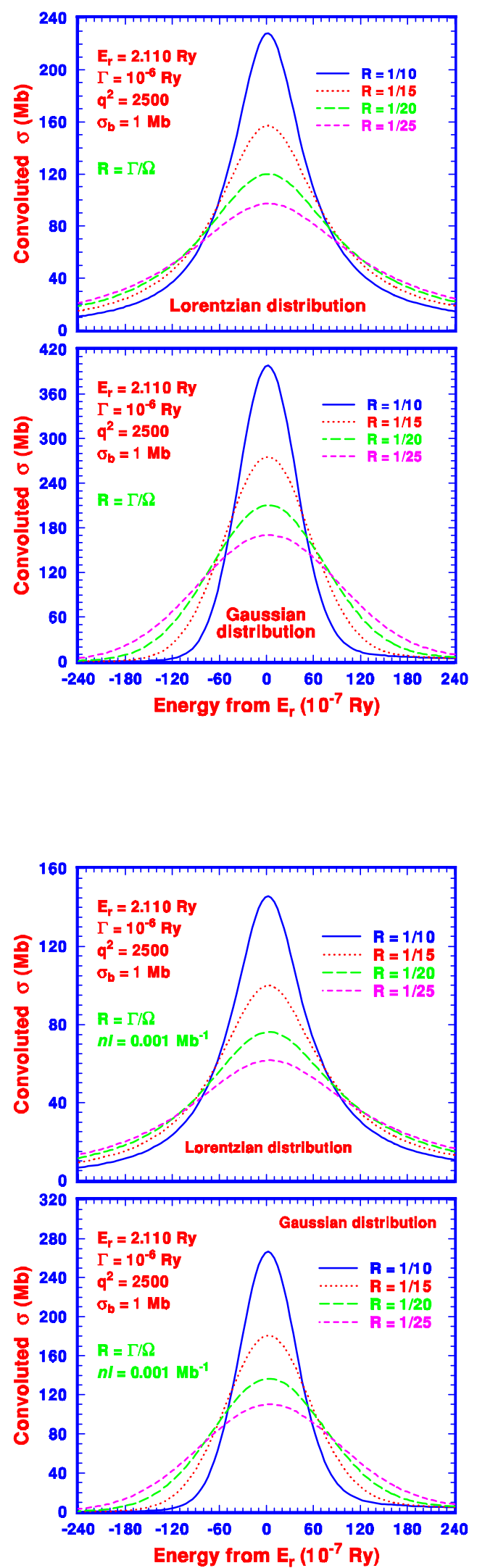

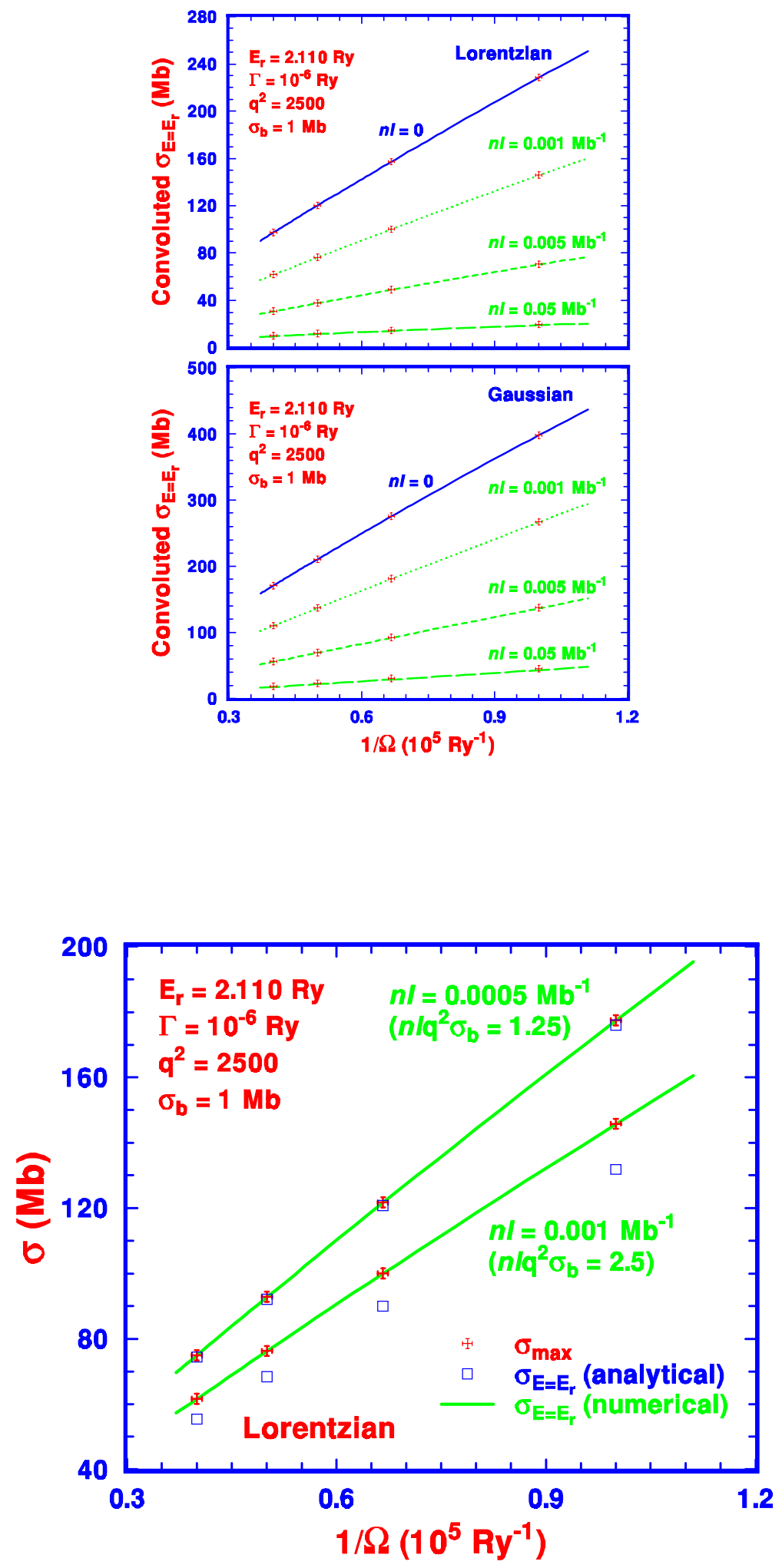


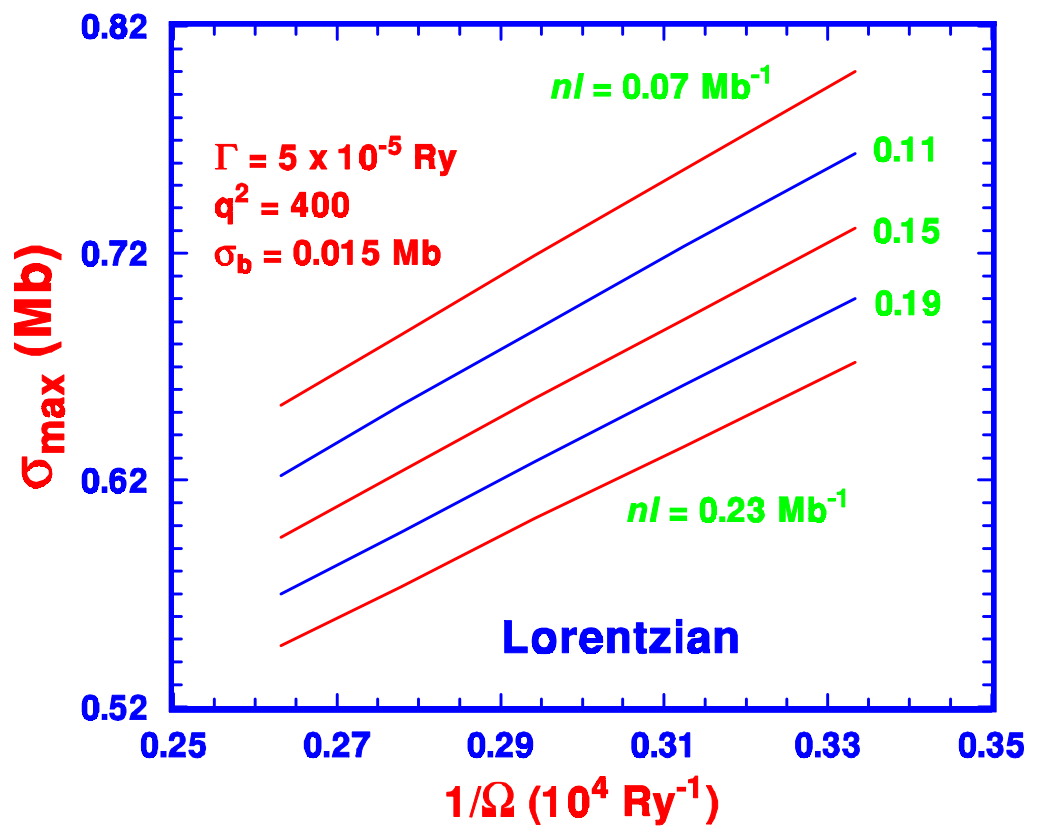

